\title{
Corela
}

Cognition, représentation, langage

HS-26 | 2018

Réseaux communautaires et variation dans le langage en moyen français et français préclassique

\section{Les enjeux de l'emploi communautaire de motifs séquenciels en analyse du discours}

\section{Pascale Mounier}

\section{CpenEdition}

Journals

Édition électronique

URL : http://journals.openedition.org/corela/6665

DOI : $10.4000 /$ corela. 6665

ISSN : 1638-573X

Éditeur

Cercle linguistique du Centre et de l'Ouest - CerLICO

Référence électronique

Pascale Mounier, « Les enjeux de l'emploi communautaire de motifs séquenciels en analyse du discours », Corela [En ligne], HS-26 | 2018, mis en ligne le 14 novembre 2018, consulté le 01 mai 2019. URL : http://journals.openedition.org/corela/6665; DOI : 10.4000/corela.6665

Ce document a été généré automatiquement le 1 mai 2019.

\section{cc) (†) ()}

Corela - cognition, représentation, langage est mis à disposition selon les termes de la licence Creative Commons Attribution - Pas d'Utilisation Commerciale - Partage dans les Mêmes Conditions 4.0 International. 


\title{
Les enjeux de l'emploi communautaire de motifs séquenciels en analyse du discours
}

\author{
Pascale Mounier
}

1 Il existe plusieurs manières d'aborder le phénomène de la variation dans le langage du point de vue de son rapport avec les communautés auquelles sont associés un variable linguistique. L'approche peut s'appliquer à la diachronie du français en mobilisant la sociolinguistique $^{1}$, l'histoire externe de la langue ${ }^{2}$ ou encore procéder de l'analyse du discours. De ce dernier point de vue la méthode des motifs séquenciels, développée ces dernières années dans le domaine de la linguistique de corpus et de la textométrie, est susceptible à notre sens de servir l'interprétation stylistique. D. Legallois, T. Charnois et T. Poibeau (2016: § 5) définissent ce qu'ils proposent d'appeler le « motif séquenciel » ou plus simplement le «motif» comme une «configuration lexico-grammaticale» récurrente. Ils utilisent la notion pour repérer des automatismes d'écriture propres à des genres ou des sous-genres textuels : tel cliché littéraire donne lieu à tel patron syntaxique impliquant des lexèmes de même classe grammaticale ${ }^{3}$. Ainsi le cliché de l'entrée en contact physique des amants dans le roman sentimental contemporain peut-il se traduire par deux réalisations linguistiques proches relevant d'un même motif séquenciel : les GN la pression de son genou contre le sien et le contact de ses lèvres contre les siennes mettent en œuvre le motif « le NC de DETPOSS NC contre le sien $»^{4}$.

2 L'intérêt de déceler des patrons flexibles partagés est de constater les phénomènes de récurrence à un plan plus large que celui de la reprise à l'identique d'unités discrètes ( genou, lèvres) ou de suites de mots (contre le sien). Le fonctionnement du motif séquenciel est cependant complémentaire de la séquence constituée de mêmes termes (contre le sien), que D. Legallois (2009) décrit comme un "segment répété "5. Le segment répété et le motif séquenciel s'avèrent en l'occurrence à notre sens deux notions opératoires pour étudier la variation linguistique plutôt que la caractérisation générique ou sousgénérique. Ils gagnent à être envisagés comme indices de reconnaissance d'un parler de groupe, dont le genre textuel est en quelque sorte une manifestation particulière. Dans la 
mesure où les choses sont assez claires en sociolinguistique, il semble utile de tâcher de dégager les implications interprétatives de l'emploi communautaire de motifs séquenciels sur le terrain de l'analyse du discours. L'étude consistera à identifier au plan théorique les enjeux du repérage du fonctionnement de structures récurrentes dans des productions écrites et orales. Elle tentera par ailleurs de vérifier à titre d'expérimentation l'efficacité de la méthode dégagée dans deux textes proches d'un point de vue littéraire et idéologique et produits dans un même milieu d'écriture. La mise au jour de l'appartenance des deux récits sentimentaux publiés vers 1540 à une communauté avec des traits de reconnaissance propres s'appuiera sur des passages à orientation argumentative, voire didactique, fonctionnant en écho.

\section{Le motif séquenciel comme révélateur d'un langage communautaire}

3 Une séquence procédant par reformulation d'une ou plusieurs séquences préexistantes met en contact des discours possédant d'une manière ou d'une autre des points communs. Elle est l'un des révélateurs de l'ouverture d'un discours oral ou écrit à d'autres discours, antérieurs ou contemporains ${ }^{6}$. Comme le segment répété le motif séquenciel se caractérise en l'occurrence par un ancrage énonciatif et social et par une portée idéologique.

\section{Ancrage énonciatif et social}

4 La suite récurrente pertinente que l'analyse du discours retient des repérages proliférants que peut obtenir le traitement automatique des textes constitue un élément reconnaissable du parler d'un ensemble de locuteurs. La structure lexico-syntaxique reprise renvoie à un réseau de sujets parlants. À la différence de 3-grammes comme il y avait ou qu'il n', repérables dans les romans d'Hugo (Legallois $2009: 48$ ) mais qui n'ont pas de singularité marquée par rapport au parler du reste de la société, d'aujourd'hui comme de l'époque, les 5-grammes du coin de l'œil et se dirigea vers la porte font par exemple référence à certains types de discours écrits seulement. Des structures peuvent en outre avoir des traits typiquement sociolectaux. Les études menées en ethnopsychologie sur le «langage de la tribu» montrent assez l'importance qu'il y a à reconnaître la part du collectif dans la production d'énoncés individuels et dans les récurrences qu'ils démontrent. Dans la perspective qui nous occupe, le motif séquenciel rapproche en tout état de cause des locuteurs plus ou moins individualisés et plus ou moins éloignés dans le temps et l'espace.

5 Deux cas d'emploi communautaire sont à envisager, selon que le segment repris renvoie à des discours oraux ou à des textes. Dans la première situation le locuteur second mobilise un fragment du langage d'un groupe, qui peut être par exemple celui des maçons ou des enseignants, ou fait communauté avec un locuteur premier ayant employé à plusieurs reprises une même suite ${ }^{7}$. Dans la seconde situation il ouvre un dialogue avec des productions écrites, que celles-ci mobilisent des langues de spécialité, enregistrées dans des dictionnaires, des traités ou encore des documents relatifs à une pratique professionnelle ${ }^{8}$, ou des textes littéraires. Avec un discours littéraire, si tant est qu'on puisse identifier la littérarité par la présence d'un sujet de fiction et d'une visée 
esthétique, la relation engage un locuteur et au moins un auteur ${ }^{9}$. Encore faut-il faire des nuances selon que l'auteur en question prend ou non en charge l'univers de fiction : celui qui produit le texte n'est pas forcé d'assumer les assertions comme le fait le locuteur d'un échange réel.

On mesure ici la différence entre l'intertextualité étroite et l'intertextualité large. Il ne s'agit pas pour le locuteur de signaler l'appartenance de son texte à une classe de textes définie par un ensemble de critères formels et thématiques, comme le font par exemple les adaptateurs et les imprimeurs de romans de chevalerie aux $\mathrm{XV}^{\mathrm{e}}$ et $\mathrm{XVI}^{\mathrm{e}}$ siècles en mobilisant le motif "NC de $\mathrm{ADJ} \mathrm{NP}$ ", où le $\mathrm{NC}$ est un hyperonyme renvoyant à la narration ${ }^{10}$. Il s'agit de créer par la reprise d'une ou plusieurs suites un réseau textuel. Cela ne préjuge évidemment pas de l'existence de rapports physiques réels entre les locuteurs : les rapports linguistiques priment sur les rapports de personnes. S'il n'est pas besoin de connaître la situation sociale des auteurs pour attester l'intertextualité puisque l'écho précis avec certains textes suffit à désigner les lectures d'un écrivain, il n'est pas non plus exclu que les structures identiques d'œuvres produites dans le même contexte spatio-temporel révèlent ou confirment l'existence d'un milieu d'écriture.

\section{Portée idéologique}

7 Autre critère de sélection du segment répété et du motif séquenciel pertinent: un substrat idéologique présent de manière évidente ou latente. La suite de termes véhicule des représentations qui entrent elles-mêmes dans un système de valeurs. En contexte argumentatif elle peut participer à des stratégies qui requièrent une analyse au plan sémantico-pragmatique. En termes psycholinguistiques, voire d'histoire des idées, elle signale la présence d'un interdiscours, c'est-à-dire d'un ensemble de formulations auxquelles l'énoncé se réfère implicitement ou non, sciemment ou non, qui le domine et à partir duquel il fait sens ${ }^{11}$. L'usage communautaire de la séquence ajoute à la notion $d^{\prime}$ '« interdiscours » le principe du sociolecte : le mot et plus encore la structure récurrents condensent la pensée du réseau social ${ }^{12}$. Il revient ainsi au récepteur et par extension à celui qui interprète le discours second d'identifier à la fois les mécanismes de polyphonie et dialogisme et de ralliement à une pensée collective dans la convocation et l'appropriation personnelle d'un ou plusieurs discours. La tâche suppose la prise en compte de l'ensemble du discours second dans la mesure où le motif séquenciel procède par économie de moyens.

La répétition d'unités discrètes ou de suites de termes identiques ou non engage un partage ou une nuance de l'opinion que celles-ci véhiculent. Presque tout se joue ici au niveau du contexte d'accueil. La reprise à l'identique d'une unité ou d'une suite peut marquer aussi bien une validation qu'une discussion de valeurs collectives. La reprise sous forme de variante peut aussi signaler une convergence ou une divergence idéologique, quoique la modification lexicale favorise la réorientation axiologique. Le premier cas trouve une illustration dans la production d'une «écriture concertée » chez des auteurs de l'entourage de Marguerite de Navarre par l'usage d'épithètes adjectivales marquant la sélection et l'unicité en association avec des substantifs du lexique religieux : des GN comme la vraie foi et la vraie piété d'un côté et le seul Dieu et la seule foi de l'autre entrent dans la production d'un langage crypté à une époque où l'évangélisme est jugé dangereux (Garnier-Mathez 2005) ${ }^{13}$. Le second est l'équivalent en termes de structure syntaxique d'une modification lexicale opérée par F. Hollande en 2013 dans une suite de 
deux termes: le GN tension amicale reformule amitié franco-allemande et couple francoallemand, présents alors dans le discours politique depuis plusieurs années, en introduisant un terme renvoyant au conflit pour pointer les difficultés diplomatiques de deux pays voisins (Garric 2015). Le contexte et les changements lexicaux sont donc essentiels dans la saisie de l'évaluation du stéréotype social.

\section{Pistes pour un mode d'étude}

9 À partir des deux niveaux de fonctionnement signifiants du segment répété et du motif séquenciel que nous avons repérés quelques pistes méthodologiques se dégagent pour l'étude des phénomènes de reprises et variations. D'abord l'interprétation doit prendre en compte la nature formelle de la séquence. Des paramètres métriques peuvent par exemple se surimposer à la chaîne syntaxique et aux classes lexicales dans la définition d'une structure récurrente. Que la récurrence se voie ou non renforcée par la structure prosodique, il faut repérer les éléments maintenus et les éléments modifiés au plan lexical d'un discours à l'autre. Il importe aussi d'évaluer le processus et le degré de figement de la stéréotypie que contribue à isoler le segment repris. Une suite a en effet un pouvoir fixateur pour le langage du groupe, spécialement quand elle est récurrente dans le discours premier. Quand elle rejoint des clichés d'expression propres à l'ensemble de la société on a affaire à une collocation, c'est-à-dire un rapprochement particulièrement fréquent de termes (la verte prairie), ou à une locution, c'est-à-dire une séquence formant un tout indissociable pour la compréhension (une faim de loup, mettre en rage) ${ }^{14}$. Dans ces cas il faut voir en quoi le patron syntaxique perd de sa flexibilité.

Ensuite on gagne à s'appuyer sur des données de fréquence. Selon la quantité forte ou faible d'occurrences de la séquence dans le discours premier la reprise sera plus ou moins sensible pour un récepteur étranger à la communauté linguistique et idéologique. Selon la quantité même d'emplois du patron dans le discours second celui-ci aura une dimension plus ou moins sociolectale. On peut aussi faire des sondages externes aux discours pour enrichir les conclusions sur la part stéréotypique et sur la part créative de la reformulation. Plus une séquence apparait dans des discours antérieurs au discours second, plus elle a un caractère figé et plus la variation lexicale a des chances d'être perçue. Quand elle connaît un pic d'emploi à une période et dans un type de discours donnés la suite est plus facilement perçue comme un marqueur d'écriture en réseau. Un sonnettiste utilisant dans les années 1650 la rime appâts / pas à la fin du sizain avec pas participant à la négation d'un verbe de sentiment se définit ainsi comme un poète mondain pratiquant une certaine lyrique amoureuse, en l'occurrence de type malherbien ${ }^{15}$.

11 Enfin on a avantage à identifier s'il existe ou non des éléments dans le contexte d'apparition de l'occurrence susceptibles d'accentuer le phénomène d'emprunt et de dialogue discursifs. Il faut voir s'il existe un interprétant signalant l'apparition du motif et jouant pour l'auditeur ou le lecteur le rôle de déclencheur mémoriel. Un commentaire métadiscursif peut par exemple venir valider ou contester l'orientation axiologique imposée par le locuteur second, comme le prouve la polémique entre certains hommes politiques français en 2013 autour de l'emploi par F. Hollande du GN tension amicale ${ }^{16}$. Selon le nombre de signaux de reformulation la séquence se placera ainsi à un point différent sur une échelle de gradualité allant de l'allusion au renvoi explicite. L'emploi d'un cliché de langue sera par corollaire plus ou moins reconnaissable en réception. 


\section{Reprise et variation lexico-syntaxiques dans deux récits sentimentaux vers 1540} mesurer sa pertinence pour la période 1400-1650, qui fait l'objet du présent du numéro, en littérature. Nous examinerons pour cela deux œuvres distinctes d'un point de vue sous-générique mais proches thématiquement : Les Comptes amoureux, recueil de nouvelles anonyme, peut-être collectif, publié dans sa version définitive vers 1542 , et Philandre, roman de chevalerie composé par Jean des Gouttes et publié en 1544. Les œuvres ont été écrites à Lyon dans un contexte d'effervescence poétique et narrative concernant la place sociale de la femme et de redéfinition théologique des principes de l'union matrimoniale ${ }^{17}$ . Il s'agit de deux récits sans antécédents directs qui ont la particularité de contenir plusieurs histoires d'amour hors mariage et de poursuivre la réflexion sur le comportement des amants menée en particulier dans des romans sentimentaux récents. Les narratrices des Comptes rapportent en effet à une assemblée le devenir d'épouses insoumises se livrant sans scrupules à leurs amants et glorifiant la résistance à la morale sexuelle dominante. Les narrateurs de premier et de second plan de Philandre légitiment pour leur part la relation adultère de Passerose et du chevalier Philandre. Outre le fait que les auteurs ont peut-être appartenu au même milieu d'écriture les textes exploitent communément le principe de la nouvelle insérée et puisent à l'occasion dans la même source $^{18}$. Nous pouvons ainsi légitimement tâcher d'identifier la nature et la portée idéologique des motifs séquenciels que l'auteur de Philandre tire des Comptes dans différents passages énonçant une déontologie amoureuse.

\section{Motifs séquenciels dans le même texte et d'un texte à l'autre}

Les suites de mots reprises dans Philandre sont souvent déjà récurrentes dans les Comptes. Plus exactement elles mobilisent des termes relevant de séries morphologiques très exploitées par les devisantes-narratrices et par l'auteur, qui prend la parole sous le pseudonyme Jeanne Flore dans une «Epistre » initiale et un « Au lecteur » final. Le verbe convenir, l'adjectif verbal convenant et le substantif convenance constituent ainsi par le mécanisme de la dérivation déployé dans l'ensemble du recueil un premier type de variation lexicale. En association avec des noms de personnages ou de personnes en relation de couple et des caractérisants désignant les propriétés de ceux-ci ils forment un second mode de répétition, de type séquenciel :

Corela, HS-26 | 2018 


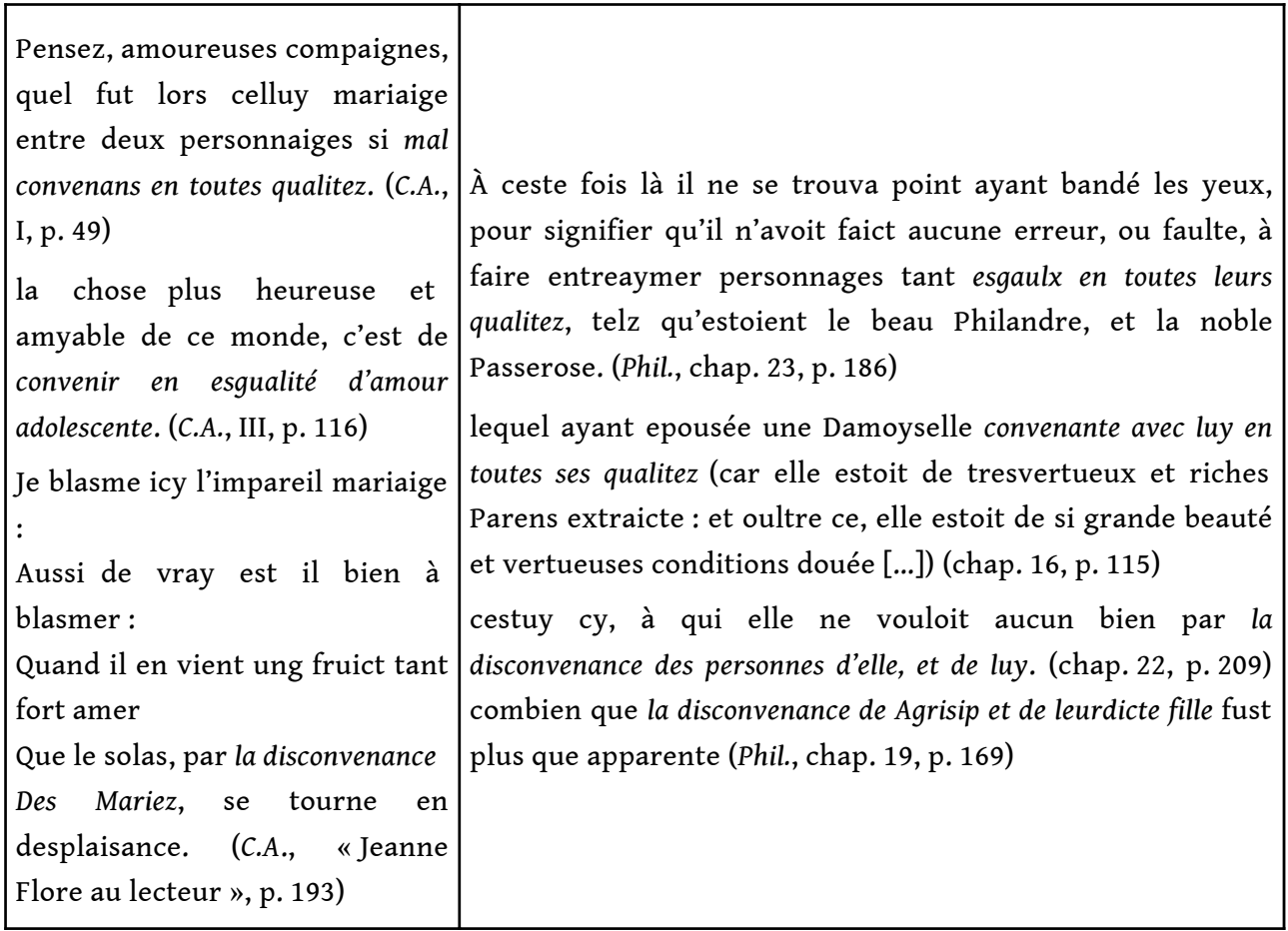

Parmi les différents patrons forgés par les Comptes exploitant le champ notionnel de l'assortiment de personnes de sexe opposé Philandre emprunte le motif «ADJ en toutes qualités » en remplaçant convenans par esgaux (1.), adjectif peut-être en lien avec le dérivé substantival esgalité du motif «V en égalité d'amour» de la nouvelle III des Comptes (2.). Dans le récit du mariage des parents de la jeune Laure, héroïne d'une nouvelle enchâssée du roman, le patron lexico-syntaxique contribue à énoncer une règle à suivre: la conformité de statut civil, d'apparence physique et de conviction morale des futurs époux. L'association des séries morphologiques de convenir et esgal, qui renvoient respectivement aux notions d'union et de conformité, fait de l'amour d'êtres de complexion identique une condition pour la réussite du couple.

15 Les formes négatives mal convenans et disconvenance soutiennent pour leur part dans les suites des exemples 1. à 3. l'idée que la disparité de nature des conjoints peut invalider $a$ contrario un engagement civil et religieux. Philandre reprend deux fois la formule polémique "la disconvenance de NC", où le NC renvoie à des personnes mariées, au poème adressé au lecteur à la fin des Comptes en l'appliquant à la situation de Passerose et du vieux Garamond ainsi qu'à celle d'Agrisippe et de Lipomène, deux époux mal assortis d'une nouvelle relatée dans le chapitre 19 (3.). Comme le GN « impareil mariage » (C.A., VI, p.162), formule phare du recueil non reprise dans le roman, le motif substantival discrédite certaines unions légales imposées. L'idéolecte commun aux récits établit ainsi que le rapprochement amoureux dépend des propriétés intrinsèques, appelés " qualitez » et " adolescen[c]e ", des individus formant un couple.

16 L'originalité des Comptes et de Philandre consiste aussi dans la réorientation sémantique de suites de mots figées. Les collocations amour fidele, constante amour, entiere amour et vray amour ou vray amant entrent en particulier en résonance dans tout recueil ${ }^{19}$. Elles viennent aussi bien du discours courtois médiéval que du langage néoplatonicien contemporain, où elles renvoient à un amour affranchi des lois sociales et plus ou moins 
épuré des préoccupations charnelles ${ }^{20}$. On retrouve dans Philandre le syntagme parfait(e) amour, qui fait tout particulièrement écho à un texte de la Querelle des Amyes ${ }^{21}$ :

\begin{tabular}{|c|c|}
\hline $\begin{array}{l}\text { ficher en vos } \\
\text { ames la vertu du } \\
\text { perfaict Amour ( } \\
\text { C.A., II, p. 85) }\end{array}$ & $\begin{array}{l}\text { "puis (dict elle) que Madame Camille treuve mercy vers vostre bonté, je ne } \\
\text { faudray à ce soir, au desceu de tout vivant, de vous conduire en sa chambre, } \\
\text { et de vous mettre entre ses excellens et amoureux bras : lors, s'il ne tient à } \\
\text { vous, vous congnoistrez de quelle parfaicte amour elle ayme. - Suffise vous, } \\
\text { dict le Gentilhomme, vostre Dame sera servie à son besoing. " (Phil., } \\
\text { chap. } 11, \text { p. } 78 \text { ) } \\
\text { "Ce faisant il me sera facile d'oublier celle, qui ne m'a jamais parfaictement } \\
\text { aymé.» (Phil., chap. } 7, \text { p. } 48 \text { ) }\end{array}$ \\
\hline
\end{tabular}

17 La collocation est insérée dans le roman dans un contexte qui détourne clairement les aspirations matrimoniales des traités, des poèmes et des récits idéalisant la femme. Le sous-entendu des propos de Philandre au sujet de la demande de Camille de le rencontrer de nuit, alors qu'ils se connaissent à peine, dénonce ainsi une certaine conception de l'amour passionnel: le chevalier corrige l'évacuation par l'abstraction de "parfaicte amour » par l'emploi de l'euphémisme «à son besoing " pour renvoyer à la relation sexuelle qu'il prévoie d'avoir avec la jeune fille. Le héros signale ailleurs l'absence de sincérité et de loyauté de son ancienne maitresse, Diane, qui l'a trompé avec d'autres amants en utilisant une variante du segment répété : le verbe aimer en structure négative modifié par parfaitement ("ne m’a jamais parfaictement aymé ») érige en idéal l'amour spontané, son apparition et son développement hors mariage. On note au passage que l'allusion infléchit l'implication largement libertine de l'occurrence des Comptes (4.) : la variante verbale de parfait amour fait entendre que les personnages ont consommé leur amour mais signale aussi que la sexualité ne suffit pas à une relation épanouie puisque l'amant se plaint du fait que la femme qu'il a aimée autrefois n'a pas su garder sa foi.

Révélée par les reprises de mots à l'identique et les motifs séquenciels, le langage du réseau que forment les Comptes et Philandre a ainsi quelque chose de crypté. Qu'ils utilisent des termes relativement originaux ou qu'ils reprennent le fonds lexical des débats contemporains de casuistique amoureuse et matrimoniale en en détournant la portée, les rédacteurs des Comptes et de Philandre forgent un idéolecte contestataire dénonçant le cadre coercitif du mariage et faisant la promotion de l'union charnelle. La complicité ou « connivence» linguistique (Garnier-Mathez 2005) des auteurs permet de définir une morale sexuelle permissive fondée à la fois sur le choix de l'aimé et la sincérité des sentiments et sur la recherche du plaisir.

\section{Éléments hétérogènes : critères fréquentiels et contextuels}

19 La fréquence d'apparition et la répartition de certaines suites dans les œuvres suggèrent toutefois des éléments d'hétérogénéité entre le discours du ou des premiers rédacteurs et celui du second. Des critères fréquentiels et contextuels permettent de fait d'affiner la conception du sentiment et des rapports entre les amants et de repérer des variations de point de vue d'une œuvre à l'autre, comme l'a déjà suggéré l'exclusion dans le discours de Philandre de la recherche de la pure concupiscence de la conception de l'amour libre (4.). 
Philandre reprend peu l'isotopie figurée de la religion pour glorifier l'amour passion. Dans les Comptes celle-ci est au contraire omniprésente. L'amant éconduit Pyrance parle des sacrifices qu'il a faits en l'honneur du « sainct nom » de Vénus (C.A., II, p. 98), tandis que le poème liminaire $\mathrm{d}$ '《 Egine Minerve aux nobles Dames amoureuses » fait l'éloge $\mathrm{du}$ « sainct service / D'amour» (C.A., p. 44). Dans le GN saint service d'amour et ses variantes ${ }^{22}$ et le segment répété sainct Amour (C.A., I, p. 46, p. 58, 82, 83, 83 ; II, p. 86 ; IV, p. 129, 130 ; VII, p. 192) le substantif Amour est employé comme nom commun par antonomase et comme désignateur rigide pour renvoyer aux dieux Cupidon et Vénus, qui interviennent dans plusieurs épisodes mythologiques des nouvelles. Absente des romans sentimentaux contemporains traduits de l'italien ou de l'espagnol, qui ont réintroduit à partir de 1525 dans la littérature française les thèmes du primat des sentiments sur les codes sociaux et de la liberté des sujets dans le choix de l'être aimé, l'association antithétique des filières lexicales du sacré et du païen ainsi que du divin et de l'humain dans des suites plus ou moins séquentielles a donc une portée ouvertement polémique.

21 Le roman de Des Gouttes reprend seulement à une occasion un terme voyant de la métaphore filée de la religion, l'adjectif composé à valeur intensive sacrosainct, en l'appliquant au substantif flambe, dont le sens est lexicalisé depuis longtemps par glissement métaphorique et métonymique :

\begin{tabular}{|l|l|}
\hline $\begin{array}{l}\text { madame Cebille commença son acerbe } \\
\text { accusation à l'encontre de la sacrosaincte divinité } \\
\text { d'Amour (C.A., I, p. 46) }\end{array}$ & $\begin{array}{l}\text { «rendre lors en ce temps l'hommaige deuë à } \\
\text { pacrosaincte flambe d'Amour » (Phil., chap. 17, } \\
\text { p. 139) }\end{array}$ \\
\hline
\end{tabular}

Les passages mis en dialogue utilisent le motif séquenciel «la sacrosainte NC d'amour » (5.), avec la même tension entre figement et défigement de la référence à l'être païen ${ }^{23}$. Chez la seule narratrice des Comptes opposée à l'amour et chez le jeune Sclarion, compagnon de Philandre destiné à prendre la place de celui-ci auprès de la belle Camille, les occurrences se placent dans des contextes argumentatifs opposés. La leçon que Sclarion adresse à Camille en commentant le refus par Garamond d'aimer alors qu'il était encore jeune conteste la position de Cébille tout en suggérant peut-être avec celle-ci le caractère forcé de l'image de la sacralisation de l'amour.

La résistance à certains aspects de la déontologie du recueil apparait plus clairement dans l'emprunt rare et contextuellement réorienté des nombreux motifs séquenciels mobilisant les filières lexicales du mépris et de la punition. Les Comptes inventent en effet des suites de mots marquant l'idée d'une révérence obligée des jeunes gens, spécialement des femmes, aux êtres qui les désirent. L'attitude d'individus s'opposant aux sentiments qu'on leur témoigne est énoncée par exemple par le verbe despriser complété par la collocation vray amour (6.), tandis que la revendication d'une sanction pour celle-ci passe par l'emploi à trois reprises, en particulier dans le sous-titre du recueil, du segment « la punition que faict Venus de ceulx qui contemnent et mesprisent le vray Amour » (7.) : 


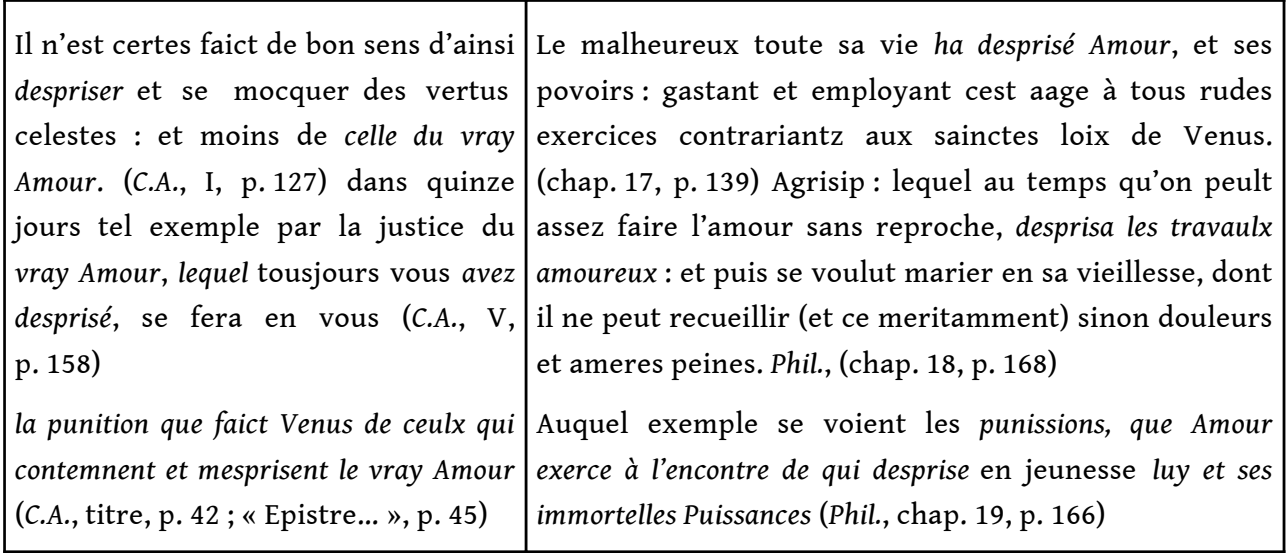

24 Philandre ne reprend pas les patrons lexico-syntaxiques de ce type, sauf au sujet de jeunes filles forcées à épouser un mari repoussant ${ }^{24}$. Sclarion et Carminian condament en particulier le mariage sur le tard voulu par les vieillards libidineux que sont Garamond et Agrisippe. Le refus d'aimer qui a concerné un temps ces maris est dénoncé sur un ton aussi violent que celui qui fait intervenir les suites de mots clés des Comptes. Les modalisateurs évaluatifs «malheureux ", " gastant » et «meritamment » et la formule à valeur déontique « on peult assez faire l'amour sans reproche » insistent sur le critère du jeune âge dans le choix des époux (6.). Il en va de même pour l'adjonction du syntagme prépositionnel à valeur temporelle «en jeunesse » au segment répété du sous-titre du recueil dans l'intertitre du chapitre 19 (7.). Alors que les Comptes mettent en avant le châtiment violent de l'insoumission à l'amour en général, Philandre soutient le refus de l'assortiment de jeunes filles et de vieillards. La présence de la notion de jeunesse dans le contexte d'emploi des champs associatifs de la désobéissance et de la sanction judiciaire suggère une méfiance à l'égard de la passion forcée. La variation opérée dans la reprise du motif séquenciel exprime l'idée que le désir vécu comme un principe irrépressible auquel il faut se soumettre est finalement aliénant car le sujet risque de perdre sa liberté.

Philandre dénonce ainsi en quelque sorte par la fréquence et le contexte d'insertion des motifs séquenciels qu'il emprunte aux Comptes en utilisant des lexèmes proches les limites de la doctrine exubérante énoncée par la narratrice principale et par les devisantes du recueil. La conflictualité argumentative établie dans les prises de parole où interviennent les suites de mots les plus polémiques du roman permet de contester certains points de la loi amoureuse érigée en norme dans l'œuvre qui les a créées ou adaptées deux ans plus tôt. Si l'on ne peut se risquer à en déduire les positions idéologiques des auteurs euxmêmes, sous peine de nier leur liberté à imiter sérieusement ou plaisamment l'idéolecte qu'ils forgent, on peut en déduire que les patrons récurrents construisent des positions idéologiques en partie distinctes. Conciliant l'idéalisation chevaleresque de l'amour et les codes sociaux du mariage, le roman de Des Gouttes refuse la réciprocité imposée de l'amour, qui conduit à la transformation de l'individu aimé en objet de plaisir; il la présente comme aussi asservissante que le lien conjugal et l'obéissance aux dieux.

26 L'étude de la portée expressive de l'emploi de suites syntaxiques de lexèmes de même série morphologique dans deux textes en fonctionnement intertextuel étroit publiés à Lyon dans les années 1540 amène à conclure au bénéfice de l'exploitation en diachronie large du repérage des motifs séquentiels selon que la définition qu'en donne le TAT. La méthode que nous proposons aide à identifier des récurrences pertinentes en analyse du 
discours parmi la multiplicité des relevés obtenus par recherche informatique. Elle consiste à repérer des enjeux interprétatifs à l'emploi communautaire d'un motif séquenciel en incitant en définitive à examiner quatre aspects conjointement :

- le rapport à la langue en général, pour évaluer le degré de figement de la séquence et la nature plus ou moins stéréotypée de l'idée véhiculée dans son emploi communautaire

- la fréquence, pour mesurer le caractère plus ou moins massif de la récurrence dans l'un et l'autre discours

- les reprises et changements lexicaux intervenant du discours premier au discours second, ce que D. Legallois, T. Charnois et T. Poibeau (2016: § 52) appellent la « variabilité» du motif, pour identifier les implications sémantiques de ceux-ci

- le contexte d'insertion, étroit et large, pour percevoir l'orientation idéologique affectée respectivement par le locuteur premier et le locuteur second à la séquence ou les interprétants signalant son emploi et trouver d'autres points de convergence, par exemple thématiques, entre les discours.

C'est donc la variation sous toutes ses formes, interne et externe, du motif séquenciel qui doit être prise en compte pour mettre au jour la volonté d'un locuteur d'entrer en interaction avec un groupe par la reprise d'un segment de son langage. La méthode, qui renouvelle l'approche du cliché, est facilement exploitable parce qu'elle s'applique à un objet simple, requérant un outillage conceptuel léger. Elle a en outre l'avantage de réconcilier des disciplines variées en sollicitant sans exclusive des repérages de différentes obédiences théoriques: énonciative, lexicologique, sémantico-pragmatiques et d'histoire de la langue.

\section{BIBLIOGRAPHIE}

Textes

Anonyme, Comptes amoureux, s. 1. n. d. [Lyon, Denis de Harsy, c. 1542] ; Reynolds-Cornell, Régine (éd.) (2005), Les Contes amoureux, Saint-Étienne, Presses de l'Université de Saint-Étienne.

Des Gouttes, Jean, Le premier livre de la belle et plaisante histoire de Philandre, surnommé le Gentilhomme, Prince de Marseille : Et de Passerose, fille du Roy de Naples, Lyon, Jean de Tournes, 1544 ; Mounier, Pascale (éd.) (2015), Philandre, Paris, Classiques Garnier, « Textes de la Renaissance ».

Études

Amossy, Ruth et Herschberg Pierrot, Anne (1997 [3ème éd. 2011), Stéréotypes et clichés, Paris, Armand Colin.

Boerm, Michael Lloyd (2008), « Pourquoi 'Pas': The Socio-Historical Linguistics Behind the Grammaticalization of the French Negative Marker », thèse de l'université du Texas (Austin).

Branca-Rosoff, Sonia, Fournier, Jean-Marie, Grinshpun, Yana et Régent-Susini, Anne (dir.) (2011), Langue commune et changements de normes, Paris, Champion, 2011. 
Dion, Laetitia (2017), Histoires de mariage. Le mariage dans la fiction narrative française (1515-1559), Paris, Classiques Garnier.

Garnier-Mathez, Isabelle (2005), L'Épithète et la connivence. Écriture concertée chez les Évangéliques français (1523-1534), Genève, Droz.

Garric, Nathalie (2015), « Polémique métalangagière sur une (dé)nomination : le terme confrontation ", in Stabilité et instabilité dans la production du sens : la nomination en discours, dir. J. Longhi, Langue française, $\mathrm{n}^{\circ} 188$, p. 5-14.

Kawaguchi, Yuji, Minegishi, Makoto et Viereck, Wolfgang (dir.) (2011), Corpus-based Analysis and Diachronic Linguistics, Amsterdam / Philadelphia, John Benjamins.

Kemp, William (2003), « La contribution lyonnaise à la diffusion d'un hispanisme grammatical sous François Ier. La comparative en non pas ", in Lyon et l'Illustration de la langue française à la Renaissance, dir. G. Defaux, Lyon, É.N.S. Éditions, p. 109-145.

Kerbrat-Orecchioni, Catherine (1997), L'Énonciation. De la subjectivité dans le langage, Paris, Armand Colin.

Kraif, Olivier, Novakova, Iva et Sorba, Julie (2016), « Constructions lexico-syntaxiques spécifiques dans le roman policier et la science-fiction ", in Phraséologie et genres de discours, Lidil, $n^{\circ}$ 53, p. 143-159.

Krieg-Planque, Alice (2012), Analyser les discours institutionnels, Paris, Armand Colin.

Larrivée, Pierre (2014), « Reanalysis of negatives as polarity markers? The last 400 years of decline of the French preverbal negative clitic », Lingua, $\mathrm{n}^{\circ} 147$, p. 40-49.

Legallois, Dominique, Charnois, Thierry et Poibeau, Thierry (2016), « Repérer les clichés dans les romans sentimentaux grâce à la méthode des 'motifs' ", Lidil, n 53, 2016, p. 95-117.

Legallois, Dominique (2009), «À propos de quelques n-grammes significatifs d'un corpus poétique du XIXe siècle », L'Information Grammaticale, n 121/1, 2009, p. 46-52.

Milroy, Lesley (1987), Language and Social Networks, New York, Blackwell.

Mounier, Pascale (2014), « Des récits d'amours libres et sensuelles au XVIe siècle : variations lexicales à Lyon vers $1540 »$, Neophilologus, vol. 98, n³, p. 385-404.

Mounier, Pascale (2014), « Une construction syntaxique en emploi sociolectal entre 1535 et 1545 : la négation renforcée dans les propositions comparatives », in Le Style, découpeur du réel, dir. L. Bougault, J.-F. Castille et L. Himy-Piéri, Rennes, PUR, « Interférences », 2014, p. 209-225.

Mounier, Pascale (2010), « Les clichés du discours anti-matrimonial vers 1540. Reprise et détournement de syntagmes figés », in « Mariage des corps, mariage des esprits » dans la littérature française de la Renaissance à l'Âge classique, dir. C. Chervet et L. Dion, site GRAC ( http://grac.univ-lyon2.fr/mariage-des-corps-mariage-des-esprits-lyonseptembre-2009--532715.kjsp?RH=grac67).

Polguère, Alain (2015), « Non-compositionnalité : ce sont toujours les locutions faibles qui trinquent », Verbum, $n^{\circ} 37 / 2$, p. 257-280.

\section{NOTES}

1. La théorie des réseaux sociaux de L. Milroy (1987), forgée à partir de l'étude des traits phonologiques de l'anglais irlandais, établit que les communautés auxquelles appartient un 
individu déterminent les variantes que celui-ci utilise. Elle ouvre des pistes stimulantes pour expliquer certains phénomènes. L'influence du catalan par mobilité géographique d'individus permet ainsi de comprendre la généralisation de pas comme forclusif de la négation (Larrivée 2014, Boerm 2008) et la création de la négation renforcée en comparative d'inégalité au XVIe siècle (Kemp 2003).

2. Sur les changements en diachronie et les variations d'une ère linguistique à l'autre en ancien français voir Kawaguchi, Minegishi et Viereck (dir.) (2011) ; sur le XVIIe siècle voir Branca-Rosoff, Fournier, Grinshpun et Régent-Susini (dir.) (2011).

3. Sur la fertilité de l'étude des clichés de style en littérature voir Amossy et Herschberg Pierrot (1997 : 53-85).

4. Kraif, Novakova et Sorba (2016) repèrent pour leur part des structures hiérarchiques ou « arbres de dépendance », qui ne correspondent pas forcément à des séquences linéaires, propres au roman policier et à la science-fiction contemporains.

5. Le segment répété se traduit en traitement automatique des textes par la notion de «ngramme ", qui désigne "toute suite de $\mathrm{n}$ formes graphiques non séparées par un délimiteur » (Legallois 2009: 46). La méthode fait par exemple apparaître les 2-grammes quelque chose et a fait ou encore les 3-grammes dans l'ombre et de la mer dans Les Contemplations et La Légende des siècles d'Hugo. Appliquée à un-sous genre et un registre traités par des auteurs différents, elle vise à identifier des critères de généricité. D. Legallois signale ainsi le segment répété je suis le comme propre à la lyrique romantique.

6. A. Krieg-Planque (2012: 186-189) rappelle les notions mobilisées habituellement par différentes disciplines pour étudier la porosité et la circulation discursives : la «polyphonie » et le «dialogisme», forgés par Bakhtine et propres aux études littéraires, l'«interdiscours", renvoyant surtout à des phénomènes de soumission idéologique et relevant du champ de l'analyse du discours, l'«intertextualité », que les littéraires utilisent pour étudier la présence d'un ou plusieurs textes dans un autre, et le " prédiscours ", renvoyant en sociolinguistique aux savoirs et aux croyances partagés mobilisés inconsciemment par le locuteur.

7. On peut prendre l'exemple du corpus des slogans de la campagne politique de François Hollande en 2012 pour accéder à la Présidence de la République, étudié par A. Krieg-Planque (2012: 196). La séquence Interdire les licenciements, c'est maintenant utilisée par un représentant d'une organisation syndicale après les élections mobilise un motif forgé à partir de phrases à détachement formulées par le candidat Hollande, comme La parité, c'est maintenant et Le mariage pour tous, c'est maintenant.

8. Sur les enjeux de la constitution d'une langue juridique dans la fondation du common law anglais en dépit du déclin de l'anglo-normand voir la contribution de C. Laske dans le présent numéro. I. Bretthauer montre ici pour sa part les mécanismes d'imitation et de singularisation dans le code graphique mis en œuvre par le Normand Guillaume Flambart dans sa copie de textes relatifs à la réglementation de l'usage des forêts. Le phénomène de la variante graphique, qui implique un écart par rapport à une norme géolinguistique ou sociolinguistique, se couple dans ce cas à celui de la variation linguistique.

9. On identifiera le titre Le ravissement de l'homme Veinstein donné par un journaliste à un article sur un écrivain émergeant appelé Alain Veinstein comme producteur d'un motif séquentiel par rappel du titre d'un roman de $\mathrm{M}$. Duras, pour citer à nouveau un exemple d'A. Krieg-Planque (2012: 194-195).

10. Quand Rabelais intitule son second roman La vie inestimable du grand Gargantua il utilise le patron syntaxique de titres comme L'histoire du vaillant chevalier Pierre de Provence et de la belle Maguelonne et Le romant de la belle Helayne de Constantinople en remplaçant les NC de sens générique par le quasi-synonyme vie.

11. Les récits journalistiques relevant du fait divers criminel produits au XIXe siècle ont par exemple pour interdiscours les traités et comptes rendus des médecins aliénistes contemporains. 
12. K. Kerbrat-Orecchioni (1997: 203) propose d'appeler un «idéolecte» une «compétence propre à un ensemble d'individus appartenant à la même communauté idéologique ». Elle forge le néologisme pour la linguistique énonciative à partir des termes idiolecte, dialecte et sociolecte. 13. La négation explétive renforcée en proposition subordonnée comparative d'inégalité, probablement importée en français à la suite de la captivité de François Ier en Italie, prend de même un caractère idéolectal entre 1535 et 1545 , avant de se propager à l'ensemble de la langue littéraire jusque vers 1650 (Kemp 2003). Les productions narratives lyonnaises et plus largement le milieu des lettrés passés à Lyon ont joué un rôle important dans son élaboration et sa diffusion à la cour (Mounier 2014).

14. A. Polguère (2015) fait un point utile sur la question complexe de la compositionnalité ou non-compositionnalité sémantique de trois catégories dont la structure lexicale et syntaxique est contrainte : les locutions, les collocations et les «clichés linguistiques » ou expressions de type phraséologique.

15. Voir l'étude menée par Miriam Speyer dans le présent numéro sur le phénomène de la variation lexico-phonique, qui montre les enjeux de filiation littéraire induits par la reprise d'une rime forgée par Malherbe (appâts / pas) chez certains poètes de la première partie du XVIIe siècle.

16. C. Bartolone, président de l'Assemblée nationale, conteste alors le fait que le Président ait employé un euphémisme pour caractériser les relations entre la France et l'Allemagne en suggérant le remplacement de la série lexicale créée autour d'amitié et ses dérivés par un NC seul dénotant le conflit : « il faut remplacer tension amicale par confrontation » (Garric 2015).

17. Sur les controverses juridiques et théologiques relatives à l'institution du mariage et leurs enjeux dans les romans et nouvelles de la Renaissance, voir Dion 2017. Nous citerons ensuite les œuvres dans les deux éditions critiques modernes disponibles (Reynolds-Cornell (éd.) 2005, Mounier (éd.) 2015).

18. Une des trois nouvelles de Philandre traduit un passage de Mambriano de Francesco Bello qu'adapte aussi la nouvelle I des Comptes (Mounier (éd.) 2015 : 64-68). Les œuvres entretiennent une relation intertextuelle privilégiée du point de vue lexical par le fait que Philandre utilise à l'identique ou sous forme de quasi-synonymes des lexèmes de la nouvelle I non seulement dans le chapitre 19 correspondant mais aussi dans l'ensemble de ses vingt-quatre chapitres (Mounier 2014).

19. Voir par exemple « vray amour» (C.A., II, p. 85 et p. 112), « entiere amour » (C.A., VI, p. 177) ou « vrais, entiers, ne loyaulx amants » (C.A., I, p. 47) et « fidele et loyal amant » (C.A., II, p. 86).

20. On les trouve aussi dans les Angoysses amoureuses, les Epistres familieres et invectives et Le Songe d'Hélisenne de Crenne (Mounier 2010).

21. La parfaicte amye d'Antoine Héroët, publié en 1542, qui a lancé la polémique en soutenant une position néoplatonicienne.

22. Elles contiennent le NC service et le NC amour ou son dérivé adjectival amoureux : "vray service d'amour » (C.A., « Epistre... », p. 45) et « devot et sacré service amoureux » (C.A., I, p. 82).

23. Voir aussi l'unique reprise du GN « sainctes loix de Venus » (Phil., chap. 17, p. 139).

24. Le motif littéraire de la mal mariée, en vogue depuis la fin du XVe siècle, a un rôle important dans Philandre (Mounier (éd.) 2015 68-74). Il apparaît dans deux des trois nouvelles insérées, aux chapitres 18 et 19, ainsi que dans l'histoire du mariage forcé de Passerose à Garamond de la fin du roman, dans les chapitres 20 à 24 . Les nouvelles en question, qui rapportent les déboires d'une jeune fille mariée à un vieillard impuissant, réfléchissent en miroir la situation de Passerose, Philandre et Garamond. 


\section{RÉSUMÉS}

La linguistique de corpus a fait avancer ces dernières années l'étude du cliché linguistique en mettant au point des techniques de repérage et de calcul de fréquence de suites syntaxiques à classes de mots identiques. Ce qu'elle appelle le «motif séquenciel» (Legallois, Charnois et Poibeau 2006) peut trouver une pertinence en analyse du discours en particulier quand il sert de révélateur d'un langage communautaire forgé par des locuteurs en relation idéologique. C'est ce que confirme la présence de motifs séquenciels à lexèmes de même série morphologique dans deux récits sentimentaux publiés à Lyon dans les années 1540. Les formules reprises à l'identique ou sous forme de variante dans les Comptes amoureux et Philandre participent à l'énoncé d'une morale amoureuse défendant de manière polémique la liberté des sujets et l'union charnelle. Ce type de récurrences constitue une manière stimulante d'aborder le phénomène de la variation linguistique dans son interaction avec un réseau communautaire.

Corpus linguistics has developed methods to identify and quantify various types of collocative phenomena. One such phenomena is the "sequential motif" (Legallois, Charnois and Poibeau 2006). Focusing on recurrent collocations forming key expressions greatly enhance literary discourse analysis. They help detect communities of writers and the ideological values that they share. Such sequential motifs are found in sentimental narratives published in Lyon in the 1540s. Recurrent collocations in the Comptes amoureux and Philandre propose a polemical defense of amorous and sexual freedom. They thus reveal values shared by the community of authors.

\section{INDEX}

Mots-clés : Suite de mots, segment répété, variante, série morphologique, cliché linguistique, linguistique de corpus

Keywords : Collocation, repeated segment, variant, morphological series, linguistic cliché, corpus linguistics

\section{AUTEUR}

\section{PASCALE MOUNIER}

Université de Caen Normandie, EA 4256 LASLAR 